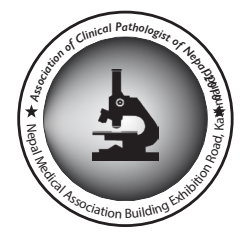

\title{
Histopathology of important fungal infections - a summary
}

\author{
Arnab Ghosh ${ }^{1}$, Dilasma Gharti Magar ${ }^{1}$, Sushma Thapa ${ }^{1}$, Niranjan Nayak², \\ OP Talwar ${ }^{1}$ \\ ${ }^{I}$ Department of Pathology, Manipal College of Medical Sciences, Pokhara, Nepal. \\ ${ }^{2}$ Department of Microbiology, Manipal College of Medical Sciences, Pokhara, Nepal.
}

\section{Keywords: \\ Fungus; \\ Mycosis; \\ Opportunistic; \\ Systemic}

\begin{abstract}
Fungal infections due to pathogenic or opportunistic fungi may be superficial, cutaneous, subcutaneous and systemic. With the upsurge of at risk population systemic fungal infections are increasingly common. Diagnosis of fungal infections may include several modalities including histopathology of affected tissue which reveal the morphology of fungi and tissue reaction. Fungi can be in yeast and / or hyphae forms and tissue reactions may range from minimal to acute or chronic granulomatous inflammation. Different fungi should be differentiated from each other as well as bacteria on the basis of morphology and also clinical correlation. Special stains like GMS and PAS are helpful to identify fungi in tissue sections.
\end{abstract}

\section{Correspondence: \\ Dr Arnab Ghosh, MD \\ Department of Pathology, \\ Manipal College of Medical Sciences, Pokhara, Nepal. \\ ORCID ID: 0000-0002-8566-2067 \\ Email:docarnab2k@yahoo.com}

Reveived : March 15t 2019 ; Accepted :March14 ${ }^{\text {th }} 2019$; Published : March $29^{\text {th }} 2019$

Citation: Ghosh A, Gharti Magar D, Thapa S, Nayak N, Talwar OP. Histopathology of important

fungal infections - a summary. J Pathol Nep 2019;9: 1490-6. DOI 10.3126/jpn.v9i1.23377

Copyright: This is an open-access article distributed under the terms of the Creative Commons Attribution 4.0 International License, which permits unrestricted use, distribution, and reproduction in any medium, provided the original author and source are credited.

\section{INTRODUCTION}

Fungal infections or mycoses may be caused by pathogenic fungi which infect healthy individuals or by opportunistic fungi in immunocompromised individuals, the latter constituting the major group. Mycoses are encountered more frequently due to increasing proportion of at risk population in the society. At risk populations for opportunistic or disseminated fungal infections include those under chemotherapy or immunosuppressive treatment, post-transplant patients, premature infants, the elderly, debilitating illness and infections like HIV. ${ }^{1}$

Diagnosis of fungal infections can be carried out by different approaches which include clinical features, demonstration of fungi and fungal culture, histopathological examination of tissue and other techniques e.g., detection of fungal antigen by enzyme immunoassay, direct fluorescence antibody, immunohistology, in situ hybridization, PCR etc. Clinically several fungal infections may mimic infections of other microbial agents. ${ }^{1,2}$ Other more sophisticated 
techniques may not be available for all types of fungi and in all centers especially in the developing countries. Histopathology though not a substitute for fungal culture can often give a presumptive or specific diagnosis if the offending fungus can be identified in the tissue sections. Histopathological diagnosis of fungi may need knowledge about fungal morphology in tissue and various reactions of the tissue in response to the infection as well as proper clinical correlation. ${ }^{3}$

To identify and assess the morphology of fungus in tissue sections, special stains are better than the routine hematoxylin eosin (H\&E) stain. Morphological parameters which may help to differentiate different types of fungi on histology include estimation of size, the predominant form of the fungus (yeast, hyphae or pseudohyphae), presence or absence of capsule, thickness of the capsule, budding (single or multiple, narrow or wide based), presence or absence of septae and branching pattern in hyphae etc. ${ }^{3}$ Morphologically fungi may grow as yeast forms, hyphae (molds) and dimorphic forms. In yeast forms, the individual fungi are seen either in short chains or are individually separated. They are round to oval and reproduce by budding. In candida species, the buds often do not detach from each other and thus form a chain of elongated yeast cells which are known as pseudohyphae. Hyphae or molds are multicellular thread like filaments which can grow and divide at the tips. Hyphae may produce conidia which can disseminate through air. Dimorphic fungi can be present in either forms. They show yeast form in human body temperature and mold forms in room temperature. ${ }^{2,3}$

\section{TISSUE REACTIONS}

The tissue harboring the infection may be of diverse types viz., skin, subcutaneous tissue and deeper organs like lung, central nervous system, bone, liver etc., and accordingly the mycoses are divided into different types. Changes in the tissue as a reaction to the infection may include allergic reaction, acute and / or chronic reaction, granuloma formation, necrosis and vascular invasion. ${ }^{1}$

Fungi secrete adhesive molecules to adhere to the tissues as well as enzymes to destroy and invade into the tissue. Usually lymphocytes and macrophages are activated to cause chronic inflammation. In some cases neutrophils also become activated and associated active inflammation is seen. Anti gen presenting macrophages secrete interleukin-12 which activates $\mathrm{CD} 4$ positive $\mathrm{T}$ helper lymphocytes. The lymphocytes secrete Interferon-Y which transforms macrophages into epithelioid cells which aggregate to form granuloma. Necrosis seen in fungal infection may be due to the ongoing inflammation or may be due to infarction of tissue if the vessels are invaded by fungal infection. Caseous necrosis mimicking tuberculosis may also be seen in some fungal infections. There also can be in vivo deposition of amorphous eosinophlic hyaline material around the fungi, which is due to local antigen antibody reaction and is known as Splendore Hoeppli phenomenon. It is often seen in sporotrichosis, entomophthoramycosis aspergillosis, blastomycosis, and also in mucormycosis. This is however quite nonspecific and may be seen in many other fungal as well as several bacterial and parasitic infections. ${ }^{2-4}$

\section{USEFUL STAINS}

Hematoxylin and eosin stain is the routine stain used in histopathology. However, though it may stain some fungi in tissue sections, it is often not adequate especially if only a few fungi are present. However this stain demonstrates the tissue reactions including inflammation, granuloma, necrosis and Splendore Hoeppli phenomenon and thus may give a clue to the pathologist. ${ }^{2}$ The main two special stains for fungi are Gomori's methanamine silver (GMS) and periodic acid Schiff (PAS) stains. The GMS is also commonly known as silver stain and stains most fungi black to brown. However GMS may also stain filamentous bacteria. PAS stains most fungi magenta. Though PAS does not stain filamentous bacteria but stain many other structures. Both GMS and PAS cannot show the associated reactive tissue response. GMS with $\mathrm{H} \& \mathrm{E}$ counterstain can demonstrate both fungi and tissue reactions. ${ }^{3}$ Mucicarmine and alcian blue are useful to stain polysaccharide capsule of Cryptococcus neoformans and India ink preparation demonstrate the same as negative staining in cerebrospinal fluid. Masson Fontana is useful to detect noncapsulated forms of Cryptococci due to its melanin content. ${ }^{2}$ Giemsa stain demonstrates Pneumocystis spp., and differentiate protozoa Leishmania from fungal infections. Acid fast stains may be helpful to distinguish filamentous Nocardia spp., which is weakly positive from fungi which are negative. ${ }^{1,2}$

Fungal infections, depending on the site and extent of the infection, can broadly be divided into superficial mycosis, cutaneous and subcutaneous mycosis and systemic infection. Candida spp. can cause cutaneous as well as systemic disease and will be discussed under the systemic group.

\section{SUPERFICIAL MYCOSES}

Superficial mycoses are confined to the outermost layers of skin or its appendages (e.g., hair). These superficial infections usually can be diagnosed clinically and its histological examination is not necessary. Patients usually seek medical opinion because of cosmetic purposes. The important infections under this category include black piedra (Piedraria hortai), white piedra (Trichosporon beigelii), both of which involve hairs and tinea versicolor (Malassezia furfur) which involve stratum corneum.,

\section{CUTANEOUS MYCOSES}

Cutaneous mycoses or dermatophytoses are caused by dermatophytes which include several fungi under the genera Epidermophyton, Microsporum and Trichophyton. ${ }^{6}$ 
All of them usually involve skin, hair and nail and these infections are commonly known as tinea or ringworm. Depending on different sites the infections are called as Tinea capitis, Tinea corporis, Tinea cruris, Tinea pedis and Tinea unguium. ${ }^{2,5}$ Clinically, the skin infections present as circular ring shaped patches in hairy skin area. Alopecia of the infected area may be seen. Vesicles and pustules formations are rare. ${ }^{5}$ On histopathology the fungi appear as hyaline septate hyphae. Associated tissue reactions include epidermal changes like hyperkeratosis and acanthosis of the epidermis. The inflammation is usually mild and shows mononuclear lymphocytic infiltrate and rarely, is of suppurative or granulomatous nature. Sometimes Splendore Hoeppli material may be present. ${ }^{3,6}$

\section{SUBCUTANEOUS MYCOSES}

The next group includes the fungi causing cutaneous and subcutaneous mycoses and the important entities are chromoblastomycosis, sporotrichosis, rhinosporidiosis, mycetoma and entomophthoramycosis. The fungi those which can cause systemic disease as well as cutaneous mycosis will be discussed under systemic group E.g., Candida sp.

Chromoblastomycosis is an indolent cutaneous infection seen more commonly in tropical countries. They are caused by several pigmented or dematiaceous fungi which cannot be differentiated on histopathology. ${ }^{2,3}$ Clinically they present as a nodule or verrucous plaque usually on extremities. Satellite nodules may be present. Histopathology reveals round thick-walled dark brown $(5-12 \mu \mathrm{m})$ fungi which are grouped mostly in dermis. ${ }^{3}$ Septations may be seen. These are known as sclerotic bodies or copper penny bodies. All different species look similar. Epidermis shows hyperkeratosis and pseudoepitheliomatous hyperplasia and the dermis shows granulomatous and suppurative inflammation with microabscess formation. Transepidermal elimination of pigmented sclerotic bodies may be seen. ${ }^{7-9}$

Sporotrichosis is caused by Sporothrix schenckii, a dimorphic fungus which are found worldwide and spread by implantation, rarely inhalation. They usually cause "lymphocutaneous disease" and only in rare cases may cause systemic disease. Typically the presentation is a solitary nodule in exposed part of the body which may ulcerate, or may discharge pus. The nodule may become verrucous if the ulcer or pus discharge continues for long duration..$^{1,2}$ Involvement of draining lymphatics may be clinically seen as chains of nodules. ${ }^{3}$ On histopathology, these fungi are spherical to oval, or even cigar shaped yeasts measuring $2-10 \mu \mathrm{m}$. Budding of the yeasts may be seen which is narrow based or tube like and is usually single and rarely multiple. ${ }^{3}$ This morphology of Sporothrix schenckii may be mimicked by Candida glabrara, Histoplasma and Leishmania. ${ }^{1}$ Epidermis shows hyperkeratosis, acanthosis and verrucous hyperplasia and the inflammation is mixed suppurative and granulomatous in nature. ${ }^{3}$ Asteroid bodies i.e., Splendore Hoeppli material arranged in star like fashion around the fungus, though not pathognomonic of sporotrichosis, may be seen in upto $92 \%$ of cases. Asteroid bodies and granuloma should not be confused with sarcoidosis. ${ }^{1,10}$ Sporotrichosis can be associated with other infections like lupus vulgaris. ${ }^{11}$

Rhinosporidium seeberi causes mainly mucosal and cutaneous infections and is endemic in India, Sri Lanka and parts of Africa. ${ }^{3}$ The usual clinical presentation is friable bulky polyp in nasal cavity, nasopharynx or palate. The other less common sites are larynx, conjunctiva, genitalia and skin. ${ }^{2} \mathrm{R}$ seeberi cannot be isolated on synthetic media, so histopathology is important for its diagnosis. ${ }^{1}$ On microscopy, immature and mature sporangia of $\mathrm{R}$ seeberi are seen in the stroma of the polyp. Immature forms are of $10-100 \mu \mathrm{m}$ size, which develop into the mature form of $100-350 \mu \mathrm{m}$. Sporangia have thin wall $(3-5 \mu \mathrm{m}$ thickness) and contain many sporangiospores $(6-8 \mu \mathrm{m}){ }^{3}$ Mature sporangiospores contain globular eosinophilic inclusion. Tissue reaction is nonspecific and show chronic or granulomatous inflammation. ${ }^{1,12}$

Mycetoma, also known as Maduramycosis or Madura foot is more seen in the tropical countries. It can be of 2 types viz., actinomycotic, caused by groups of filamentous bacteria (actinomycetes) and eumycotic, caused by several fungi (eumycetes) e.g., Madurella spp. Differentiating these two types is important as their treatments are different. ${ }^{2}$ Actinomycotic infections are treated with antibiotics while eumycotic infections require surgical debridement. The infection in the cutaneous and subcutaneous tissue often also involves the underlying bone. Clinically it presents as swollen "tumor like" lesions with multiple sinuses with serosanguinous granular discharge. Granules can be of varying sizes and colors like white, yellow, black and are basically compact mycelial aggregate. ${ }^{3}$ The clinical appearance and the erosion of the underlying bone on radiology may lead to erroneous diagnosis of a tumor., ${ }^{2,3}$ On histopathology, eumycotic mycetoma are composed of septate and branched fungal hyphae which are broad 2-6 $\mu \mathrm{m}$ in width. The actinomycotic mycetoma are composed of bacterial filaments which are delicate, branched, beaded and only $1 \mu \mathrm{m}$ in width. The bacterial filaments are Gram positive. Surrounding tissue shows abscess formation, Splendore Hoeppli material deposition, granulation tissue with marked edema. ${ }^{2,3}$

Entomophthoramycosis is caused by fungal agents belonging to genera Conidiobolus and Basidiobolus and is a rare chronic subcutaneous infection that may affect immunocompetent hosts predominantly in tropical and subtropical regions. They also may cause infection of sinuses and respiratory system. ${ }^{2}$ In tissue, they present as hyphae with transverse septae and the surrounding tissue shows Splendore Hoeppli material with chronic inflammation. They are often morphologically similar to and so have 
to be differentiated from mucormycosis which is a more aggressive disease. Entomophthoromycosis is characterised by lack of vascular invasion, frequent septae and presence of Splendore - Hoeppli phenomenon surrounding the hyphae and mucormycosis are aseptate or sparsely septate hyphae, vasculocentric and Splendore - Hoeppli phenomenon are relatively unusual. ${ }^{2,13}$

\section{SYSTEMIC MYCOSIS}

Systemic infection comprises the most important group from the clinical point of view. Systemic and disseminated mycoses are usually seen as opportunistic infections. High risk group includes immunocompromized patients e.g., long term steroid use, HIV/AIDS, malignancy especially hematological malignancy, post chemotherapy, renal transplant, bone marrow transplant as well as patients with long term broad spectrum antibiotics, morbid obesity, diabetes (especially if uncontrolled), burn, dialysis, cardiac valve replacement etc. ${ }^{14,15}$ With the advent of modern treatment modalities, the overall duration of survival in seriously ill patients e.g., transplant recipients, post chemotherapy, those with long term dialysis etc. have prolonged than the past resulting in an upsurge in the at risk group for mycoses in the population. ${ }^{1}$

Candida species are benign commensal in skin, mouth, gastrointestinal tract and vagina. If the skin or mucosal barrier is breached, then it may cause infection. ${ }^{2}$ Conditions like vaginitis and diaper rash are common in healthy individuals. Patients with uncontrolled diabetes, burn, dialysis, intravenous catheters are of high risk category for more severe infection. Disseminated candida infection may be seen in neutropenia, leukemia, chemotherapy, bone marrow transplant and in AIDS. ${ }^{3}$ The commonest species are Candida albicans. Normal host response includes recruitment of neutrophils and macrophages which lead to phagocytosis and oxidative killing of the infective fungi. The filamentous forms of fungi may escape phagocytosis if there is neutropenia and / or defect in the process of phagocytosis e.g., defect in NADPH oxidase, myeloperoxidase. They yeast forms of fungi produce $\beta 1,3$, glucan which activates dendritic cells. The dendritic cells produce interleukin 6 and 23 which activate the T lymphocytes leading to further recruitment of neutrophils and monocytes. Low $\mathrm{T}$ cell count (as in HIV infection) or ineffective T cell may cause disseminated infection. Virulence of the fungus is related to the increased adherence to host cells. Fungi secrete integrin which binds to fibrinogen, fibronectin and laminin and agglutinins which binds to epithelial cells. Invasiveness is the ability of the fungus to invade the tissue. The enzymes aspartyl proteinase helps to degrade extra cellular matrix and catalase helps to resist the oxidative killing by macrophages. ${ }^{3,4,16}$ Oral thrush is superficial mucosal infection of the oral cavity and presents as grey white pseudomembrane composed of matted organism and inflammatory debris. If the membrane is removed, the underlying hyperemia and inflammation are revealed. Oral thrush is seen in newborn, debilitated or immunocompromised person and with long use of oral steroid and antibiotics. ${ }^{2}$ Candidal esophagitis is presents as painful swallowing usually in patients with AIDS and hematological malignancy. Pseudomembrane is seen on endoscopy. Females with diabetes, pregnancy, contraceptive pills are prone to candidal vaginitis which presents as intense itching and "curd-like" thick discharge. ${ }^{3}$ Cutaneous candidiasis can affect different sites and present as various forms onychomycosis, paronychia, folliculitis, intertrigo, balanitis , diaper rash etc. ${ }^{2,5}$ Invasive candidiasis is seen when candida infection is disseminated through blood stream. Invasive infection may present as abscess formation in various organs such as kidney, myocardium, endocardium, brain and meninges, eye, liver and often seen in patients with neutropenia or chemotherapy. ${ }^{14,15,17}$ Histopathology of the affected tissue reveals candida as oval budding yeast forms $(2-6 \mu \mathrm{m})$, or as pseudohyphae which are chain of budding yeasts or rarely, as true hyphae. They can be visualized with H\&E, GMS and PAS stains. ${ }^{3}$ The degree and type of inflammation in the tissue are widely varied ranging from neutrophil abscess and suppurative inflammation, granuloma formation to minimal inflammation in neutropenic patients. ${ }^{2,3}$ Candida may invade vessels leading to ischemic necrosis or infarction of the involved tissue as well as mycotic aneurysm and thrombophlebitis of the vessels. ${ }^{17}$

Aspergillosis is the second most common opportunistic mycoses. The commonest species is A. fumigatus. They are transmitted as airborne conidia. The mostcommonly involved organs are lungs, nasal cavity and sinuses., ${ }^{2,3}$ Several other sites, though rare, are reported to be involved. ${ }^{18}$ In healthy persons, it causes allergic bronchopulmonary aspergillosis while in immunocompromised patients it may cause serious sinusitis, aspergillus pneumonia, aspergilloma, invasive pulmonary aspergillosis, and disseminated aspergillosis. ${ }^{1,3}$ Aspergilloma is a brownish fungus ball composed of matted tangled Aspergillus hyphae seen in a lung cavity due to tuberculosis, bronchiectasis or an old abscess. ${ }^{2}$ Asperigillus on microscopy presents as hyphae with conidia. The hyphae are uniform, narrow (5-10 $\mu \mathrm{m})$, non-pigmented (hyaline), regularly septated and branching at acute angle (40 degree). On H\&E stain, viable hyphae appear basophilic and degenerated and necrotic hyphae appear eosinophilic. These hyphae are strongly positive with PAS and GMS. ${ }^{3}$ Splendore Hoeppli material may be seen. If there is fungal invasion of the vessels, infarct and necrosis of the tissue may be seen. ${ }^{2,3,18}$ Similar hyphae on histology can be seen with Fusarium spp., Scedosporium spp., Trichoderma spp., Paecilomyces spp., and Mucorales genera. Mucorales have non-pigmented (hyaline) pauci-septate ribbon like hyphae with right angle branching. ${ }^{1}$

Cryptococcosis include infection by Cryptococcus species which comprise of C. neoformans and C. gattii, the latter being discovered relatively recently in 1999. Cryptococci are present in the nature in soil, bird dropping (especially pigeon) and trees. ${ }^{2,3} \mathrm{C}$. neoformans is responsible for majority of the 
infections found in immunocompromised individuals while C. gattii causes infections in immunocompromised and competent hosts. C. gatti is more limited in geographical distribution than C. neoformans. ${ }^{1}$ Patients with HIV infections are particularly at risk. Other predisposing conditions include malignancies (especially Hodgkin's lymphoma), defective cellular immunity, autoimmune diseases and diseases involving lungs, liver and kidney.,19 The fungus has a polysaccharide capsule which inhibits phagocytosis by alveolar macrophages, leukocyte migration and recruitment of inflammatory cells. Enzymes secreted by the fungus include serine proteinase which helps in tissue destruction and invasion and mannitol dehydrogenase which produces mannitol leading to edema. ${ }^{2}$ Cryptococcus also possesses a unique enzyme phenol oxidase which catalyzes conversion of L dehydrophenylalanine (L-DOPA) to melanin. Melanin is an antioxidant, protects the organism from intracellular killing by phagocytic cells as well as counteracts antifungal agents, binds iron and provides cell wall integrity to the fungus. ${ }^{3}$ The infection spreads through inhalation and cryptococci are neurotropic. So, the major organs involved are lung and central nervous system. ${ }^{2}$ Lung infection or cryptococcal pneumonia is usually symptomless. ${ }^{4}$ In central nervous system meninges, grey matter, basal nuclei are mostly affected and fungi are seen in the cerebrospinal fluid. ${ }^{19}$ Cerebromeningeal cryptococcosis is usually fulminant and if untreated, mostly fatal. ${ }^{1,3}$ Some cases may show subacute or chronic course producing small cyst like spaces in the gray matter, the so called "soap bubble" lesions, which contain numerous fungi. ${ }^{2}$ Other organs like liver, spleen, adrenal, bone can be involved in severe immunocompromised patients. ${ }^{1}$ On microscopy the fungus shows only yeast forms (3-5 $\mu \mathrm{m})$ with narrow based budding. The yeasts have a characteristic thick gelatinous polysaccharide capsule which is positive with Alcian blue and mucicarmine. As with any other yeasts, the wall of the organism stains with PAS and GMS stains. ${ }^{3,19,20}$ To identify the yeasts in the CSF, India ink preparation can be used as a negative stain to highlight the thick capsule in a dark background. ${ }^{2,3,19}$ However, rarely, Cryptococci may have less prominent capsule or absent capsule. In tissue, Cryptococci are positive with Masson Fontana stain for melanin and it can be helpful to identify noncapsulated strains. ${ }^{2,19}$ These strains, on histology, may be confused with Candida spp., and HIstoplasma. ${ }^{1}$ Tissue reaction widely varies from minimal inflammation to granulomatous inflammation. It is to be noted that cryptococcal antigen testing in serum or CSF depends on detecting antigens present in the capsule and may be negative in poorly encapsulated strains. ${ }^{21}$

Mucormycosis is caused by fungi belonging to the family Mucormycetes, which are widely distributed in the nature (soil, dust). They infect mainly immunocompromised patients. They are transmitted usually via inhalation of spores causing infection of nasal sinuses and lungs and rarely through ingestion causing involvement of gastrointestinal tract. ${ }^{22}$ Clinically mucormycosis may present as rhinitis, pneumonia or cerebral infection. Pneumonia is usually seen in immunocompromised patients and cerebral infection involving brain and orbit is seen in uncontrolled diabetes. ${ }^{23}$ On microscopy, they are non-septate, broad $(6-50 \mu \mathrm{m})$ hyphae of variable width with right angle branching. Folding of hyphae may be seen. Vascular invasion and tissue destruction can be present. ${ }^{1,3}$

Histoplasma capsulatum is present in soil especially with bird and bat droppings. Inhalation of dust and soil with microconidia causes the infection. ${ }^{3}$ They are intracellular pathogens, found in macrophages (similar to mycobacteria). Clinically histoplasmosis may mimic tuberculosis. It may cause primary pulmonary infection which is usually a self-limiting disease, secondary chronic progressive pulmonary infection, infections of extra-pulmonary sites e.g., adrenal, liver, meninges and even disseminated disease in immunocompromised patients. ${ }^{2,24}$ Chest $\mathrm{X}$ Ray may reveal lesions mostly in upper lobe which often mimics tuberculous lesion. Associated changes in the lung like consolidation, cavity formation, calcification and fibrosis may occur. Histopathology shows macrophages with many intracellular thin walled small yeasts $(3-5 \mu \mathrm{m})$. The yeasts are pear shaped with narrow based budding., ${ }^{3,25}$ On tissue sections, small variants of B dermatitidis, capsule deficient Cryptococcus, endospores of Coccidioides spp., $\mathrm{P}$ jiroveci, Penicillium marneffei and Candida glabrata can be confused. ${ }^{1,25}$ Tissue reaction is very similar to tuberculosis with epithelioid granuloma and caseous necrosis. ${ }^{24}$

Blastomycosis is caused by Blastomyces dermatitidis which is a soil inhabitating dimorphic fungus. ${ }^{1}$ Inhalation of conidia is the usual route of infection. Clinically, it may present as various respones involving mainly lungs ranging from asymptomatic infection to fatal acute respiratory distress symptoms. Pulmonary blastomycosis may present with clinical features similar to tuberculosis. ${ }^{26}$ Chest Xray shows involvement of the upper lobe mostly and the lesions mimic tuberculosis or even a neoplasm. ${ }^{1,3}$ Depending on extent of the disease there may be lobar consolidation, multiple nodules and even miliary infiltrates. It also may cause disseminated blastomycosis, and rarely, primary cutaneous blastomycosis. ${ }^{26}$ Fungi appear as yeast cell (5 $15 \mu \mathrm{m}$ in size), thick double-contoured cell wall, visible multiple nuclei and may show single broad-based bud. Surrounding tissue often shows necrotising granuloma mimicking tuberculosis. On HE stain, the capsule appears as a clear space around the fungi and can be stained by GMS and PAS stains. ${ }^{3}$ However, endospores of Coccidioides spp., Candida spp., Histoplasma, Cryptococcus and Aspergillus conidia can mimic on histology. ${ }^{1}$

Pneumocystis species are widely present in warmblooded vertebrates and has both fungal and protozoan characteristics. ${ }^{1}$ P. jiroveci is specific for humans. It causes opportunistic infection only in immunocompromised patients, especially in AIDS patents. Commonest organ 
involved is lung. Clinically, pneumocystis pneumonia is a type of interstitial pneumonia which is progressive and fatal if not treated. ${ }^{27}$ Characteristically, patients present with dry nonproductive cough. Alveoli become filled with eosinophilic pink foamy material giving the appearance like "honey comb". This foamy material is composed of fungi and cell debris. Trophozoites and cyst forms are seen in lung alveoli. Trophozoites are described as cup or boat shaped, 1- $4 \mu \mathrm{m}$ in size, neucleated and stain as pale blue with Giemsa stain. Cysts are round or indented, 6-8 $\mu \mathrm{m}$ in size, and stain well with GMS. ${ }^{3}$ The surrounding tissue usually show minimal inflammation. In case of extrapulmonary pneumocystis infection, it can be confused with Histoplasma. $^{1}$

Infection by Coccidioides immitis is due to inhalation of arthroconidia leading to delayed hypersensitivity reaction. ${ }^{28}$ Conidia inhibits phagocytosis. Clinically, most are asymptomatic. Only $1 \%$ present as disseminated disease. In immunocompromised patients various organs like lungs, meninges, skin, bones, adrenals, lymph nodes, spleen, liver can be affected. ${ }^{2}$ This fungi appear as spherules within macrophages or giant cells. These sperules are 10 to $100 \mu \mathrm{m}$, thick-walled, non-budding and often filled with multiple small endospores ( 2 to $5 \mu \mathrm{m}){ }^{3}$ On fungus morphology, the differentials include Blastomyces, HIstoplasma, Candida, Pneumocystis. R seeberi also show sporangia with endospores which are larger ${ }^{29}$ Usually there is granuloma formation in the tissue mimicking tuberculosis. If the spherules rupture, it may lead to suppurative reaction. ${ }^{2}$

Paracoccidioidomycosis is caused by a dimorphic fungus P. brasiliensis and occurs mainly in South America as an endemic disease. It mainly affects males as it is suggested that $17-\beta$ estradiol blocks or delays formation of pathogenic yeast forms from the mycelial or conidial forms. ${ }^{30}$ Majority of patients resent with pulmonary infection. The course of the disease varies and is usually more acute in children and more chronic in adults. Wide spread dissemination may occur. ${ }^{2}$ On tissue sections, the yeasts are of varying sizes $(5-60 \mu \mathrm{m})$ with a surrounding clear space. The yeasts show characteristic multiple budding which is described as "Micky Mouse ears" or as "ship steering wheel", if the budding is circumferential., 30 The yeasts are usually within giant cells but can also be extracellular. The host reactions may include granuloma formation, necrosis and neutrophilic inflammation. ${ }^{1,3,30}$

Any mycosis has to differentiated from bacterial infections especially nocardiosis, actinomycosis, botryomycosis which may mimic filamentous fungi on histology but will require antibiotics and / or surgical debridement as treatment. ${ }^{31}$ Nocardiosis is caused by thin filamentous aerobic bacteria Nocardia spp. (e.g., $\mathrm{N}$ asteroids), which are positive with Gram stain and weak positive with acid fast stains. Major sites which may be involved by nocardiosis include lung and central nervous system. ${ }^{2}$ Actinomycosis is caused by a group of filamentous anaerobic bacteria (e.g., Actinomyces israelii) which may present as abscesses in different sites as already mentioned above. On tissue sections they appear as aggregates composed of delicate branched filaments of $1 \mu \mathrm{m}$ width are often surrounded by Splendore Hoeppli material. They are positive with Gram stain but negative with Ziehl Neelsen stain. ${ }^{1,2}$ Botryomycosis is rare and is caused by non-filamentous Gram positive or negative nonfilamentous bacteria. ${ }^{3,31}$

Histopathology of the infected tissue to identify the causative fungus needs adequate knowledge of their morphology and availability of special stains which helps to highlight them and to differentiate them not only from each other but also from other nonfungal structures. The associated tissue reactions sometimes may be the first clue to the diagnosis. Lastly, the histopathologist should also possess ample clinical knowledge so that appropriate correlation with clinical data of the patient can be carried out which is a must before the final diagnosis.

\section{Conflict of Interest: None}

\section{REFERENCES}

1. Guarner J, Brandt ME. Histopathologic diagnosis of fungal infections in the 21st century. Clin Microbiol Rev. 2011;24:247-80. Crossref

2. Chander J. Textbook of medical mycology. 4th ed. New Delhi: Jaypee; 2018.905p

3. Chandler FW, Watts JC. Fungal Diseases. In: Damjanov I, Linder J, editors. Anderson's Pathology. 10th ed. St. Louis : Mosby;1996.pp 951-62.

4. McAdam AJ, Milner DA, Sharpe AH. Infectious diseases. In: Kumar V, Abbas AK, Aster JC. Editors. Robbins and Cotran Pathologic Basis of Disease. Ninth edition. Philadelphia, PA: Elsevier, 2015. pp.341-402.

5. Hinshaw MA, Longley JB. Fungal Diseases. In: Elder DE, Rosenbach M, Murphy GF, Rubin AI, Xu X. editors. Lever's histopathology of the skin. 11th ed. Philadelphia: Wolters Kluwer; 2015. pp. 72760 .

6. Surendran K, Bhat RM, Boloor R, Nandakishore B,Sukumar D. A clinical and mycological study of dermatophytic infections. Indian J Dermatol. 2014;59:262-7. Crossref

7. Pradhan SV, Talwar OP, Ghosh A, Swami RM, Shiva Raj KC, Gupta S. Chromoblastomycosis in Nepal: a study of 13 cases. Indian J Dermatol Venereol Leprol. 2007;73:176-8. $\underline{\text { Crossref }}$

8. Sayal SK, Prasad G K, Jawed K Z, Sanghi S, Satyanarayana S. Chromoblastomycosis. Indian J Dermatol Venereol Leprol 2002;68:233-4. Crossref

9. Queiroz-Telles F, Esterre P, Perez-Blanco M, Vitale RG, Salgado CG, Bonifaz A Chromoblastomycosis: an overview of clinical manifestations, diagnosis and treatment. Med. Mycol 2009;47:3-15. $\underline{\text { Crossref }}$

10. Rodríguez G, Sarmiento L. The asteroid bodies of sporotrichosis. Am. J. Dermatopathol. 1998;20:246-9. 당ssref

11. Dwari BC, Ghosh A, Paudel R, Kishore P. A clinicoepidemiological study of 50 cases of cutaneous tuberculosis in a tertiary care teaching hospital in Pokhara, Nepal. Indian J Dermatol 2010;55:233-7. $\underline{\text { Crossref }}$

12. Capoor MR, Khanna G, Rajni, Batra K, Nair D, Venkatchalam VP, Aggarwal P. Rhinosporidiosis in Delhi, North India: case series from a non-endemic area and mini-review. Mycopathology 2009;168: 89-94. Crossref 
13. Das P, Vijay MK, Joshi P, Yadav R, Singh G. Histological identification of Entomophthoromycosis in biopsy samples is required. Indian J Pathol Microbiol. 2014;57:514-6 $\underline{\text { Crossref }}$

14. Concia E, Azzini AM, Conti M. Epidemiology, incidence and risk factors for invasive candidiasis in high-risk patients. Drugs. 2009:69(Suppl.1);5-14. Crossref

15. Darouiche RO. 2009. Candida in the ICU. Clin Chest Med. 2009;30:287-93. $\underline{\text { Crossref }}$

16. Fidel PL Jr. History and update on host defense against vaginal candidiasis. Am J Reprod Immunol. 2007;57:2-12. Crossref

17. Sargent J, O'Marcaigh A, Smith O, Butler K, Gavin P, O'Sullivan M. Candida albicans-associated necrotizing vasculitis producing life-threatening gastrointestinal hemorrhage. Hum. Pathol. 2010; 41:602-4. Crossref

18. Riscili BP, Wood KL. Noninvasive pulmonary Aspergillus infections. Clin Chest Med. 2009;30:315-35. Crossref

19. Gazzoni AF, Severo CB, Salles EF, Severo LC. Histopathology, serology and cultures in the diagnosis of cryptococcosis. Rev Inst Med Trop Sao Paulo. 2009;51:255-9. Crossref

20. Huston SM, Mody $\mathrm{CH}$. Cryptococcosis: an emerging respiratory mycosis. Clin Chest Med. 2009;30:253-64, Crossref

21. Lin TY, Yeh KM, Lin JC, Wang NC, Peng MY, Chang FY. Cryptococcal disease in patients with or without human immunodeficiency virus: clinical presentation and monitoring of serum cryptococcal antigen titers. J Microbiol Immunol Infect. 2009;42:220-6. Crossref

22. Ribes JA, Vanover-Sams CL, Baker DJ. Zygomycetes in human disease. Clin Microbiol Rev. 2000;13:236-301. Crossref
23. Roden MM, Zaoutis TE, Buchanan WL, et al. Kontoyiannis DP, Walsh TJ. Epidemiology and outcome of zygomycosis: a review of 929 reported cases. Clin Infect Dis. 2005;41:634-53.Epub 2005 Jul 29.

24. Kauffman CA. Histoplasmosis.Clin Chest Med. 2009;30:217-25. Crossref

25. Gupta N, Arora SK, Rajwanshi A, Nijhawan R, Srinivasan R. Histoplasmosis: cytodiagnosis and review of literature with special emphasis on differential diagnosis on cytomorphology. Cytopathology. 2010;21:240-4. Crossref

26. Patel AJ, Gattuso P, Reddy VB. Diagnosis of blastomycosis in surgical pathology and cytopathology: correlation with microbiologic culture. Am J Surg Pathol. 2010;34:256-61. $\underline{\text { Crossref }}$

27. Ng VL, Yajko DM, Hadley WK. Extrapulmonary pneumocystosis. Clin Microbiol Rev. 1997;10:401-18. Crossref

28. Parish JM, Blair JE. Coccidioidomycosis. Mayo Clin Proc 2008;83:343-9. Crossref

29. Saubolle MA. Laboratory aspects in the diagnosis of coccidioidomycosis. Ann N Y Acad Sci. 2007;1111:301-14. Crossref

30. de Arruda JAA, Schuch LF, Abreu LG, et al. A multicentre study of oral paracoccidioidomycosis: Analysis of 320 cases and literature review. Oral Dis. 2018;24:1492-502.

31. Sullivan DC, Chapman SW. Bacteria that masquerade as fungi: actinomycosis/nocardia. Proc Am Thorac Soc. 2010;7:216-21. $\underline{\text { Crossref }}$ 